\title{
Factores asociados a la enfermedad de Parkinson en pacientes de la Comarca Lagunera, México
}

\author{
Raymundo Ventura-Chávez ${ }^{1}$, Ana I. Reyna-Gil² y Silvia García ${ }^{3 *}$ \\ ${ }^{1}$ Departamento de Neurología y Psicología, Unidades Médicas de Alta Especialidad, Torreón, Coahuila; ${ }^{2}$ Departamento de Medicina Interna, Hospital \\ General de Zona $n .^{\circ} 51$ Gómez Palacio, Durango, Instituto Mexicano del Seguro Social; ${ }^{3}$ Departamento de Investigación Clínica, "Centro Médico \\ Nacional 20 de Noviembre", Instituto de Seguridad y Servicios Sociales de los Trabajadores del Estado. Ciudad de México. México
}

\begin{abstract}
Resumen
Introducción: La enfermedad de Parkinson (EP) es un padecimiento neurodegenerativo progresivo, incapacitante e incurable; en el $90 \%$ de los casos es de etiología poligénica y multifactorial. La Comarca Lagunera es la novena zona más poblada de México, está ubicada en el centro-norte; aquí las concentraciones de arsénico, cadmio y plomo superan en 1-3 órdenes de magnitud los niveles aceptables. Objetivo: El objetivo de esta investigación fue evaluar la asociación de los factores ambientales en el desarrollo de la EP en esta región. Material y métodos: Se incluyeron pacientes y sujetos controles de más de 18 años que aceptaron participar firmando el consentimiento informado escrito, oriundos y residentes de la Comarca Lagunera; el diagnóstico de EP se determinó de acuerdo con los Criterios diagnósticos del Banco de Cerebros del Reino Unido; los controles fueron personas sanas y sin antecedentes de enfermedad neurológica. En todos se realizó una evaluación neurológica completa. Resultados: Se incluyeron 204 personas: 60 con diagnóstico de EP y 144 controles; encontramos que el consumo de tabaco, café y té se asociaban negativamente con el desarrollo de EP, en tanto que la ubicación de la vivienda, la fuente del agua de consumo, y el contacto con pesticidas y plomo no mostraron asociación. Conclusiones: En concordancia con otros estudios, el tabaquismo, el consumo de café y de té se asociaron negativamente al desarrollo de EP, en tanto que el consumo de agua de pozo, exposición a pesticidas y/o plomo, y tipo de vivienda rural no tuvieron asociación; así, su presunta participación como «detonadores» de la enfermedad sigue siendo controvertida.
\end{abstract}

Palabras clave: Enfermedad de Parkinson. Factores ambientales. Área de la Comarca Lagunera.

\section{Factors associated with Parkinson's disease in patients from the Comarca Lagunera, Mexico}

\section{Abstract}

Introduction: Parkinson's disease (PD) is a progressive, incapacitating and incurable neurodegenerative disease; in 90\% of cases its etiology is polygenic and multifactorial. Comarca Lagunera is the ninth most populated area of Mexico, located in

\section{Correspondencia:}

*Silvia García

Centro Médico Nacional 20 de Noviembre, ISSSTE

Departamento de Investigación Clínica

San Lorenzo, 502

Col. del Valle

Fecha de recepción: 06-07-2018

Fecha de aceptación: 28-02-2019

C.P. 03100, Ciudad de México, México

E-mail: rolasil@yahoo.com.mx E-mail: rolasil @ yahoo.com.mx DOI: 10.24875/RMN.M19000067 www.revmexneurociencia.com
1665-5044/@ 2019. Academia Mexicana de Neurología A.C. Publicado por Permanyer México. Este es un artículo Open Access bajo la licencia CC BY-NC-ND (http://creativecommons.org/licenses/by-nc-nd/4.0/).

Disponible en internet: 09-08-2019 Rev Mex Neuroci. 2019;20(4):174-179 www.revmexneurociencia.com
ss bajo la licencia CC BY-NC-ND 
the center-north; its concentrations of arsenic, cadmium and lead exceed in 1-3 orders of magnitude acceptable levels. Objective: To evaluate the association of environmental factors with the development of $P D$ in this region. Materials and Methods: Patients and controls were more of 18 years old, they agreed to participate with the signature of the informed consent; they were natives and residents of Lagunera region; PD diagnosis was made according Diagnostic Criteria of the Brain Bank of the United Kingdom; controls were healthy people without history of neurological disease. All of them underwent neurological evaluation. Results: 204 people were included: 60 persons with PD diagnoses and 144 controls; consumption of tobacco, coffee and tea were associated to negative effect to develop PD, while rural housing, source of water consumption, and the exposition to pesticides and lead were not associated to PD. Conclusions: In accordance with other studies, smoking, consumption of coffee and tea were protective factors for the development of $P E$, while consumption water well, exposure to pesticides and/or lead, and rural housing were not associated to PD; so, their presumed participation as "triggers" of the disease remains controversial.

Key words: Parkinson's disease. Environmental factors. Comarca Lagunera area.

\section{Introducción}

La enfermedad de Parkinson (EP) se considera, dentro de los padecimientos neurodegenerativos, la más frecuente después de la enfermedad de Alzheimer. Es una enfermedad progresiva, incapacitante e incurable; sin embargo, la calidad de vida y la expectativa han mejorado con los tratamientos actuales ${ }^{1}$. La prevalencia estimada de la EP es del $0.3 \%$ en población general y del $3 \%$ en mayores de 60 años. En México la prevalencia varía de 40 a 50 casos por 100,000 habitantes/ año².

En el $10 \%$ de los casos el origen es genético, de trasmisión mendeliana; la gran mayoría, el 90\%, se cataloga como EP esporádica, definida como poligénica y multifactorial. La hipótesis ecogenética explica la interacción entre ambos tipos ${ }^{3,4}$.

La Comarca Lagunera es la novena zona más poblada de México, ubicada en el centro-norte incluye parte de los Estados de Coahuila y Durango, y debe su nombre a los cuerpos de agua alimentados por los ríos Nazas y Aguanaval, ahora remplazados por las presas Lázaro Cárdenas y Francisco Zarco ${ }^{5}$. El agua tiene los siguientes usos: agrario (89\%), público urbano (7\%), pecuario (2\%) e industrial (2\%). El $60.6 \%$ se extrae del subsuelo y el resto de aguas superficiales (Comisión Nacional del Agua [CNA], 2005) 6-8. Dada la diversidad de contaminantes ambientales en la Comarca Lagunera, se debe analizar si dichas condiciones participan en la presentación de la EP 9 .

Si bien la predisposición genética es relevante en la EP los detonantes parecen ser ambientales, mismos que aún faltan por identificar ${ }^{10}$ para cada población. Los factores de riesgo reconocidos para desarrollar EP son: la edad mayor a 60 años ${ }^{11}$, el sexo (varones) ${ }^{12}$ y el efecto protector del tabaquismo ${ }^{13}$, la ingesta de café o té ${ }^{14,15}$; otros factores de riesgo menos claros son el traumatismo craneoencefálico ${ }^{16}$, el consumo de alcohol ${ }^{17,18}$, vivir en comunidades rurales, el contacto con herbicidas o pesticidas, la ingestión de agua de pozo ${ }^{19}$, la exposición a metales (manganeso ${ }^{20}$, plomo ${ }^{19}$, y arsénico ${ }^{21,22}$ ), la dieta ${ }^{23,24}$ y el uso de tamoxifeno ${ }^{25}$. El uso de levodopa y su impacto en el curso de la EP sigue siendo controvertido ${ }^{26}$.

La Comarca Lagunera es la región más afectada de México por el hidroarsenicismo agudo. En 1962 en Torreón se notificaron 40 casos graves y una defunción; actualmente se manifiesta como hidroarsenicismo crónico endémico 27,28 . Las concentraciones de arsénico, cadmio y plomo en la región superan en uno, dos y hasta tres órdenes de magnitud los niveles considerados como aceptables para sitios contaminados, lo que incrementa los riesgos de efectos adversos en la salud en las poblaciones vecinas ${ }^{11}$.

El objetivo de esta investigación fue evaluar la asociación de los factores ambientales en el desarrollo de la EP en una población del norte (Comarca Lagunera) de México.

\section{Material y métodos}

Se realizó un estudio observacional, trasversal, analítico, comparativo y abierto. La muestra de casos se obtuvo de manera consecutiva en la consulta externa del Servicio de Neurología de la Unidad Médica de Alta Especialidad n. ${ }^{\circ} 71$ del IMSS, unidad de concentración de pacientes de la Comarca Lagunera. Se incluyeron pacientes de más de 18 años, oriundos y residentes de la Comarca Lagunera, con diagnóstico de EP de acuerdo con los Criterios diagnósticos del Banco de Cerebros de la Sociedad de la enfermedad de Parkinson del Reino Unido (UKPDSBB) ${ }^{29}$ y que aceptaron participar en el estudio firmando el consentimiento informado. Las personas controles fueron acompañantes 
de las salas de espera de consulta externa y seleccionadas por aleatorización simple, sin antecedente o enfermedad neurológica y por lo demás con las mismas características demográficas que los pacientes con EP y que aceptaron participar en el estudio firmando el consentimiento informado.

En todos se realizó una evaluación médica neurológica completa. Los enfermos con EP se estratificaron de acuerdo con la Unified Parkinson's Disease Rating Scale (UPDRS) ${ }^{30}$. El análisis de la información fue procesado mediante el SPSS ${ }^{\circledR} 15$, se realizó estadística descriptiva y razón de Momios (RM) e intervalo de confianza (IC) del 95\%.

\section{Resultados}

Fueron incluidos 204 individuos, de los cuales 60 tenían el diagnóstico de EP de acuerdo con los criterios del UKPDSBB y 144 personas clínicamente sanas; todos los participantes tenían su residencia en el área de la Comarca Lagunera durante al menos 5 años en el momento del estudio; se clasificaron por grupos etarios, en décadas. En el grupo de controles se observa que son significativamente más jóvenes. Analizados por sexo, antecedente familiar de EP y tipo de vivienda no se observaron diferencias (Tabla 1). El tiempo de evolución de la enfermedad fue de 1 a 30 años, media de $7.5 \pm 6.24$ años. A excepción de la edad, los grupos eran comparables. Obtener la anuencia de personas sin EP mayores para participar en el estudio fue una limitación en este trabajo, por otro lado, y como fortaleza de la investigación, la relación casos/controles fue 1:2.4.

Se analizaron los factores asociados a la EP (Tabla 2); de este análisis destaca que el consumo de tabaco, de café y de té estuvo asociado a un efecto protector para desarrollar EP, en tanto que factores ambientales como la ubicación de la vivienda, la fuente del agua de consumo, y el contacto con pesticidas y plomo no mostraron asociación alguna.

A partir de estos datos, evaluamos si el tiempo de consumo de tabaco o la cantidad de ingesta de café 0 té se asociaron al efecto protector, encontrando que para el tabaco y la ingesta de té hubo una relación estadísticamente significativa (Tabla 3 ).

\section{Discusión}

El grupo de pacientes estudiados tenían $62.5 \pm 11.19$ años de edad, había más varones que mujeres, y más o menos una década antes les había sido diagnosticada la enfermedad (54.98 \pm 12.03$)$, datos que concuerdan
Tabla 1. Características demográficas por grupo

\begin{tabular}{|l|c|c|c|}
\hline & Casos & Controles & Total \\
\hline Edad & & & $\mathrm{p}=0.04$ \\
30 a 40 años & 3 & 24 & 27 \\
41 a 50 años & 4 & 24 & 28 \\
51 a 60 años & 15 & 34 & 49 \\
61 a 70 años & 23 & 38 & 61 \\
71 a 80 años & 12 & 21 & 33 \\
81 a 90 años & 3 & 3 & 6 \\
\hline Total & 60 & 144 & 204 \\
\hline Sexo & & & $p=0.08$ \\
Femenino & 31 & 54 & 85 \\
Masculino & 29 & 90 & 119 \\
\hline Total & 60 & 144 & 204 \\
\hline Tipo de vivienda & & & $\mathrm{p}=0.54$ \\
Urbana & 52 & 129 & 181 \\
Rural & 8 & 15 & 23 \\
Total & 60 & 144 & 204 \\
\hline Antecedente familiar de EP & 4 & 4 & $\mathrm{p}=0.19$ \\
Sí & 56 & 140 & 8 \\
\hline No & 60 & 144 & 196 \\
Total & & & 204 \\
\hline
\end{tabular}

EP: enfermedad de Parkinson.

con los estudios epidemiológicos de este padecimiento en diversas poblaciones ${ }^{31-33}$. A excepción de la edad, los controles eran comparables a los enfermos con EP. La relación casos/controles fue 1:2.4, lo que le proporciona robustez al estudio.

Se analizaron algunos factores potencialmente relacionados con la enfermedad; en nuestra investigación el hábito tabáquico se asoció negativamente a la EP (RM: 0.51; IC 95\%: 0.28-0.94; $p=0.032$ ), como se ha observado consistentemente en múltiples estudios al respecto ${ }^{34}$; aunque no hay consenso de cómo el tabaquismo protege a la enfermedad, se ha sugerido una relación biológica estrecha entre el receptor nicotínico de acetilcolina y el sistema dopaminérgico estriatal ${ }^{35}$, donde la activación de estos receptores modularía la liberación de dopamina ${ }^{36}$; además parece que la nicotina posee un efecto protector limitando el daño que produce la 1-metil-4-fenil-1,2,3,6-tetrahidropiridina en las neuronas estriatales ${ }^{37,38}$.

Como en otras investigaciones, el consumo de café también se asoció negativamente a la EP (RM: 0.64; IC 95\%: 0.51-0.95; $p=0.019)^{39,40}$, este efecto «beneficioso"se ha atribuido a una acción antagonista selectiva sobre el receptor de adenosina $A_{2 A}$ en las neuronas estriatopalidales y con ello la atenuación de la neurodegeneración dopaminérgica ${ }^{41,42}$. Análogo a otros estudios ${ }^{43,44}$, el consumo de té tuvo una relación inversa con la EP (RM: 0.64; IC 95\%: 0.48-0.85; $p=0.0001$ ). 
Tabla 2. Factores asociados a la enfermedad de Parkinson (EP)

\begin{tabular}{|c|c|c|c|c|c|}
\hline & N. ${ }^{0} \operatorname{EP}(\%)$ & N. ${ }^{0}$ controles $(\%)$ & $\mathbf{R M}$ & IC $95 \%$ & Valor de $p$ \\
\hline $\begin{array}{l}\text { Vivienda } \\
\text { Urbana } \\
\text { Rural }\end{array}$ & $\begin{array}{c}52(86.7) \\
8(13.3)\end{array}$ & $\begin{array}{c}129(89.6) \\
15(10.4)\end{array}$ & 1.2 & $0.57-2.89$ & 0.628 \\
\hline $\begin{array}{l}\text { Fuente del agua } \\
\text { Pozo } \\
\text { Potable }\end{array}$ & $\begin{array}{c}3(5) \\
57(95)\end{array}$ & $\begin{array}{c}5(3.5) \\
139(96.5)\end{array}$ & 1.4 & $0.338-6.327$ & 0.907 \\
\hline $\begin{array}{l}\text { Tabaquismo } \\
\text { Positivo } \\
\text { Negativo }\end{array}$ & $\begin{array}{l}10(16.7) \\
50(83.3)\end{array}$ & $\begin{array}{l}47(32.6) \\
97(67.4)\end{array}$ & 0.51 & $0.28-0.94$ & 0.032 \\
\hline $\begin{array}{l}\text { Alcoholismo } \\
\text { Positivo } \\
\text { Negativo }\end{array}$ & $\begin{array}{l}12(20) \\
48(80)\end{array}$ & $\begin{array}{c}19(13.2) \\
125(86.8)\end{array}$ & 0.92 & $0.80-1.62$ & 0.62 \\
\hline $\begin{array}{l}\text { Consumo de café } \\
\text { Positivo } \\
\text { Negativo }\end{array}$ & $\begin{array}{l}33(55) \\
27(45)\end{array}$ & $\begin{array}{l}52(36.1) \\
92(63.9)\end{array}$ & 0.70 & $0.51-0.95$ & 0.019 \\
\hline $\begin{array}{l}\text { Exposición a pesticidas } \\
\text { Positivo } \\
\text { Negativo }\end{array}$ & $\begin{array}{c}8(13.3) \\
52(86.7)\end{array}$ & $\begin{array}{c}12(8.3) \\
132(91.7)\end{array}$ & 1.6 & $0.69-3.71$ & 0.30 \\
\hline $\begin{array}{l}\text { Consumo de té } \\
\text { Positivo } \\
\text { Negativo }\end{array}$ & $\begin{array}{l}26(43.3) \\
34(56.7)\end{array}$ & $\begin{array}{c}26(18.1) \\
118(81.9)\end{array}$ & 0.64 & $0.48-0.85$ & 0.0001 \\
\hline $\begin{array}{l}\text { Exposición a plomo } \\
\text { Positivo } \\
\text { Negativo }\end{array}$ & $\begin{array}{c}5(8.3) \\
55(91.7)\end{array}$ & $\begin{array}{c}9(6.3) \\
135(93.8)\end{array}$ & 1.23 & $0.59-2.57$ & 0.55 \\
\hline $\begin{array}{l}\text { Uso de anticonceptivos hormonales } \\
\text { Negativo } \\
\text { Positivo }\end{array}$ & $\begin{array}{c}26(83.9) \\
5(16.1)\end{array}$ & $\begin{array}{c}50(92.6) \\
4(7.4)\end{array}$ & 0.41 & $0.103-1.683$ & 0.373 \\
\hline
\end{tabular}

RM: razón de momios.

Tabla 3. Relación de tiempo de consumo de tabaco y cantidad de café y té

\begin{tabular}{|l|c|c|}
\hline & Media & Valor de p \\
\hline $\begin{array}{l}\text { Tiempo en años de consumo de } \\
\text { tabaco } \\
\quad \text { Casos } \\
\text { Controles }\end{array}$ & $\begin{array}{c}2.32 \pm 6.703 \\
7.29 \pm 13.273\end{array}$ & 0.006 \\
\hline $\begin{array}{l}\text { Tazas estándar de té }(236.5 \mathrm{ml}) \\
\quad \text { Casos }\end{array}$ & $0.58 \pm 0.809$ & 0.001 \\
\hline $\begin{array}{l}\text { Controles } \\
\text { Tazas estándar de café }(236.5 \mathrm{ml})\end{array}$ & $0.24 \pm 0.594$ & \\
\hline $\begin{array}{l}\text { Casos } \\
\text { Controles }\end{array}$ & $0.63 \pm 0.66$ & 0.39 \\
\hline
\end{tabular}

Este efecto protector se atribuye a sus acciones antioxidante, antinflamatoria, quelante de hierro, polifenoles, a la teonina y a la cafeína.

La asociación negativa entre tabaco, café y té han sido muy consistentes en diversos estudios y diversas poblaciones, sin embargo, la asociación de EP y eventos ambientales como exposición a metales, pesticidas, herbicidas, tipo de agua de consumo, vivienda rural y microrganismos no han sido consistentes, por lo que se ha planteado la posibilidad de que dicha exposición sea en edades tempranas ${ }^{45}$ y esa sea la razón de tantas discrepancias entre las investigaciones. Nosotros proponemos que los eventos ambientales además sean diferentes en las distintas poblaciones y esta sea otra razón de por qué los resultados no se replican. Como en este estudio, donde la contaminación por metales (arsénico, cadmio y plomo) y del agua han sido documentados en la región geográfica donde se realizó la investigación y, sin embargo, no tuvieron asociación alguna con la $\mathrm{EP}^{9}$ lo cual deja en entredicho la importancia de los «detonadores» ambientales estudiados en otras investigaciones y su participación en la EP en esta población.

La explicación de la asociación de EP y metales se ha sustentado en algunas evidencias, entre ellas, en que en la sustancia negra de pacientes con EP se ha encontrado mayor cantidad de hierro, cobre y zinc que 
en controles, metales que también están presentes en los depósitos amiloides y participan en la formación de placas seniles, ovillos neurofibrilares y están implicados en los procesos de oxidación, la homeostasis del calcio y la muerte neuronal; sin embargo, en los estudios basados en cuestionarios de exposición laboral a metales no se ha hallado la asociación entre exposición laboral a plomo, cobre, hierro, mercurio, zinc 0 manganeso y el riesgo de $\mathrm{EP}^{46}$.

Los habitantes de áreas rurales están estrechamente relacionados con el consumo de agua de pozo y la exposición a herbicidas y pesticidas, que son eventos relacionados al estilo de vida. Un metaanálisis ${ }^{47}$ encontró un ligero incremento en el riesgo de desarrollar EP en habitantes de regiones rurales (Riesgo Relativo (RR): 1.17; IC 95\%: 1.10-1.24), y consumidores de agua de pozo (Riesgo Relativo RR: 1.30; IC 95\%: 1.12-1.51); en este mismo estudio concluyeron que las asociaciones positivas fueron mínimas, que las investigaciones sobre EP y la vida rural, la agricultura y el uso de pesticidas, el gradiente biológico y la temporalidad del inicio de la enfermedad no se han investigado adecuadamente. $Y$ aunque con los pesticidas había una asociación positiva consistente, los resultados carecían plausibilidad biológica, pues ni el gradiente biológico ni el periodo de latencia hasta el diagnóstico de EP después del uso de pesticidas se evaluaron adecuadamente.

Esta investigación tiene algunas debilidades que compartimos con publicaciones similares, la mayoría de nuestros sujetos de estudio $(86.7 \%$ en EP y $89.6 \%$ en controles) eran citadinos, con baja frecuencia de exposición a pesticidas (13.3\% en EP y $8.2 \%$ en controles), y aún más baja frecuencia en el antecedente de consumo de agua de pozo ( $5 \%$ en EP y $3.5 \%$ en controles). Sin embargo, a diferencia del café, el té y el tabaco, que de forma consistente están asociados negativamente a la EP, en los resultados de distintas publicaciones sobre el efecto deletéreo de un estilo de vida rural, este no ha sido consistente y el presente estudio abona en ese sentido. Habrá de considerarse que estos factores "detonadores» quizá coparticipen con variantes genéticas específicas de ciertas poblaciones y eso explique resultados diferentes.

Por otro lado, es posible que tanto los sujetos que viven en área rural como los citadinos tengan la misma exposición a los metales contaminantes de la región y que por ello no se encuentren dichas diferencias, en cuyo caso deberían verse diferencias en mayor prevalencia e incidencia de la enfermedad en esta región, que no evaluamos en esta investigación.
Otra debilidad fue que los sujetos controles era más jóvenes que los casos, esto aunque se justifica por la dificultad de obtener la anuencia de personas sin EP y mayores para participar en el estudio, debe de tenerse en cuanto a los resultados.

\section{Conclusiones}

En concordancia con otros estudios, el tabaquismo, el consumo de café y té se asociaron negativamente al desarrollo de la EP.

El consumo de agua de pozo, la exposición a pesticidas y plomo, así como la vivienda rural, no tuvieron asociación con la EP, por lo que su presunta participación como "detonadores» de la enfermedad, en concordancia con la literatura científica, sigue siendo controvertida.

\section{Financiamiento}

La presente investigación no ha recibido ayudas específicas provenientes de agencias del sector público, sector comercial o entidades sin ánimo de lucro.

\section{Conflicto de intereses}

Los autores declaran no tener conflicto de intereses.

\section{Bibliografía}

1. Varanese S, Birnbaum Z, Rossi R, Di Rocco A. Treatment of advanced Parkinson's Disease. Parkinsons Dis. 7;2010:480260. doi: 10.4061/ 2010/480260.

2. Diagnóstico y tratamiento de la Enfermedad de Parkinson inicial y avanzada en el tercer nivel de atención. Secretaría de Salud [Internet]. México: CENETEC; 2010 [fecha de consulta: 5 de junio de 2018.] Disponible en: http://www.cenetec.salud.gob.mx/descargas/gpc/CatalogoMaestro/153_GPC_PARKINSON/SSA_153_08_GRR_Parkinson.pdf

3. Douglas MR, Lewthwaite AJ, Nicholl DJ. Genetics of Parkinson's disease and Parkinsonism. Expert Rev Neurother. 2007;7:657-66.

4. Klein C, Schlossmacher MG. Parkinson disease, 10 years after its genetic revolution. Multiple clues to a complex disorder. Neurology. 2007; 69:2093-104.

5. Arriaga-Cabrera L, Espinoza-Rodríguez JM, Aguilar-Zúñiga C, Martínez-Romero E, Gómez-Mendoza L, Loa-Loza E (coord.). Regiones terrestres prioritarias de México [Internet]. México: Comisión nacional para el conocimiento y uso de la biodiversidad; 2000 [fecha de consulta: 5 de junio de 2018]. Disponible en: http://bioteca.biodiversidad.gob.mx/janium/ Documentos/1036.pdf

6. Cervantes MC, Franco González RAM. Diagnóstico ambiental de la Comarca Lagunera [Internet]. México: Facultad de Filosofía y letras, UNAM; 2010 [fecha de consulta: 5 de junio de 2018]. Disponible en: http://docplayer.es/28258983-Diagnostico-ambiental-de-la-comarca-lagunera.html

7. Flores Hernández A, Hernández F, Orona Castillo MI. Programa Integral de Transferencia de Tecnología y Servicios Agropecuarios Laguna (PITTSAL). Rev Mex Agroneg. 2004;VIII(14):252-8.

8. Santamaría J, Reta Sánchez D, Chávez González JF, Cueto Wong GA, Romero Paredes Jl. Caracterización del medio físico en relación a cultivos forrajeros alternativos para la Comarca Lagunera. Libro Técnico Núm. 2. INIFAP-CIRNOC-CELALA. 2006;2-240-

9. García Vargas GG, Rubio Andrade M, Rosales González MG, Goytia Acevedo R, García Arenas G, Candelas Rangel JL, et al. Contaminación por metales en suelos de la ciudad de Torreón Coahuila. México. Rev Chapingo ser zonas áridas. 2007;6:165-8. 
10. Varanese S, Birnbaum Z, Rossi R, Di Rocco A. Treatment of advanced Parkinson's disease. Parkinsons Dis. 2011;2010:480260.

11. Allam MF, del Castillo AS, Navajas RFC. Factores de riesgo de la enfermedad de Parkinson. Rev Neurol. 2003:36:749-55.

12. de Rijk MC, Tzourio C, Breteler MM, Dartigues JF, Amaducci L, Lopez-Pousa S, et al. Prevalence of parkinsonism and Parkinson's disease in Europe: the EUROPARKINSON Collaborative Study. European Community Concerted Action on the Epidemiology of Parkinson's disease. J Neurol Neurosurg Psychiatry. 1997;62:10-5.

13. Hernán MA, Takkouche B, Caamaño-Isorna F, Gestal-Otero JJ. A meta-analysis of coffee drinking, cigarette smoking, and the risk of Parkinson's disease. Ann Neurol. 2002;52:276-84.

14. Ascherio A, Zhang SM, Hernán MA, Kawachi I, Colditz GA, Speizer FE, et al. Prospective study of caffeine consumption and risk of Parkinson's disease in men and women. Ann Neurolo. 2001:50:56-63.

15. Barranco Quintana JL, Allam MF, Del Castillo AS, Navajas RF. Parkinson's disease and tea: a quantitative review. J Am Coll Nutr. 2009;28:1-6.

16. Kenborg L, Rugbjerg K, Lee P-C, Ravnskjær L, Christensen J, Ritz B, et al. Lassen head injury and risk for Parkinson disease: Results from a Danish case-control study. Neurology. 2015;84:1098-103.

17. Bettiol SS, Rose TC, Hughes CJ, Smith LA. Alcohol consumption and Parkinson's disease risk: A review of recent findings. J Parkinsons Dis. 2015;5:425-42.

18. Fukushima W, Miyake Y, Tanaka K, Sasaki S, Kiyohara C, Tsuboi Y, et al. Alcohol drinking and risk of Parkinson's disease: a case-control study in Japan. BMC Neurol. 2010;10:111.

19. Willis AW, Evanoff BA, Lian M, Galarza A, Wegrzyn A, Schootman M, et al. Metal emissions and urban incident Parkinson disease: a community health study of Medicare beneficiaries by using geographic information systems. Am J Epidemiol. 2010;172:1357-63.

20. Guilarte TM. Manganese and Parkinson's disease: A critical review and new findings. Environ Health Perspect. 2010;118:1071-80.

21. Cholanians AB, Phan AV, Ditzel EJ, Camenisch TD, Lau SS, Monks TK. From the cover: Arsenic induces accumulation of $\alpha$-synuclein: Implications for synucleinopathies and neurodegeneration. Oxicol Sci. 2016;153:271-81.

22. Tyler CR, Allan AM. The effects of arsenic exposure on neurological and cognitive dysfunction in human and rodent studies: A review. Curr Environ Health Rep. 2014;1:132-47.

23. Etminan M, Gill SS, Samii A. Intake of vitamin E, vitamin C, and carotenoids and the risk of Parkinson's disease: a meta-analysis. Lancet Neurol. 2005;4:362-55.

24. de Lau LM, Koudstaal PJ, Witteman JC, Hofman A, Breteler MM. Dietary folate, vitamin B12, and vitamin B6 and the risk of Parkinson disease. Neurology. 2006;67:315-318.

25. Latourelle JC, Dybdahl M, Destefano AL, Myers RH, Lash TL. Risk of Parkinson's disease after tamoxifen treatment. BMC Neurol. 2010:12:10:23

26. Clarke CE. Does levodopa therapy delay death in Parkinson's disease? A review of the evidence. Mov Disord. 1995;10:250-6.

27. Castro de EsparzaML. Presencia de arsénico en el agua de bebida en América Latina y su efecto en la salud pública [Internet]. International Congress, Ciudad de México, junio de 2006 [fecha de consulta: 6 de junio de 2018]. Disponible en: http://www.bvsde.ops-oms.org/bvsacd/ cd51/arsenico-agua.pd

28. Ochoa Reyes J, Vidal Lozano A, Lerma Santana A, Gómez Reza L, Reta Sánchez A, Fernández Escajeda D, et al. Arsenicismo subagudo y crónica en una población rural. Dermatologia Rev Mex. 2009;53:63-9.
29. T Gibb WRG, Lees AJ. The relevance of the Lewy body to the pathogenesis of idiopathic Parkinson's disease. J Neurol Neurosurg Psychiatry. 1988;51:745-52.

30. Martinez-Martin P, Prieto João Forjaz M. Longitudinal metric properties of disability rating scales for Parkinson's disease value in health. Value Health. 2006;9(6):386-93.

31. Rizek P, Kumar N, Jog MS. An update on the diagnosis and treatment of Parkinson disease. CMAJ. 2016;188:1157-65.

32. Rodríguez-Violante M, Villar-Velarde A, Valencia-Ramos C, Cervantes-Arriaga A. Características epidemiológicas de pacientes con enfermedad de Parkinson de un hospital de referencia en México. Neurocien (Mex) INNN. 2011;16:64-8.

33. Muangpaisan W, Mathews A, Hori H, Seidel D. Systematic review of the prevalence and incidence of Parkinson's disease in Asia. J Med Assoc Thai. 2011;94:749-55.

34. Ma C, Liu Y, Neumann S, Gao X. Nicotine from cigarette smoking and diet and Parkinson disease: a review. Transl Neurodegener. 2017;6:18.

35. Zhou FM, Wilson CJ, Dani JA. Cholinergic interneuron characteristics and nicotinic properties in the striatum. J Neurobiol. 2002;53:590-605.

36. Grady SR, Salminen O, Laverty DC, Whiteaker P, Mclntosh JM, Collins AC, et al. The subtypes of nicotinic acetylcholine receptors on dopaminergic terminals of mouse striatum. Biochem Pharmacol. 2007; 74:1235-46.

37. Quik M, Perez XA, Bordia T. Nicotine as a potential neuroprotective agent for Parkinson's disease. Mov Disord. 2012;27:947-57.

38. Ascherio A, Schwarzschild MA. The epidemiology of Parkinson's disease: risk factors and prevention. Lancet Neurol. 2016;15:1257-72.

39. Costa J, Lunet N, Santos C, Santos J, Vaz-Carneiro A. Caffeine exposure and the risk of Parkinson's disease: a systematic review and meta-analysis of observational studies. J Alzheimers Dis. 2010;20(Suppl 1):S221-38.

40. Schwarzschild MA, Agnati L, Fuxe K, Chen JF, Morelli M. Targeting adenosine A2A receptors in Parkinson's disease. Trends Neurosci. 2006;29:647-54

41. Gyoneva S, Shapiro L, Lazo C, Garnier-Amblard E, Smith Y, Miller GW, et al. Adenosine A2A receptor antagonism reverses inflammation-induced impairment of microglial process extension in a model of Parkinson's disease. Review Neurobiol Dis. 2014;67:191-202.

42. Prediger RD. Effects of caffeine in Parkinson's disease: from neuroprotection to the management of motor and non-motor symptoms. J Alzheimers Dis. 2010;20(Suppl 1):S205-220.

43. Feng-Jiao Li, Hong-Fang Ji, Liang She. A meta-analysis of tea drinking and risk of Parkinson's disease. Neurobiol Dis. 2014;67:191-202.

44. Qi H, Li S. Dose-response meta-analysis on coffee, tea and caffeine consumption with risk of Parkinson's disease. Geriatr Gerontol Int. 2014; 14:430-9.

45. Logroscino G. The role of early life environmental risk factors in Parkinson disease: what is the evidence? Environ Health Perspect. 2005: 113:1234-8

46. Wirdefeldt K, Adami HO, Cole P, Trichopoulos D, Mandel J. Epidemiology and etiology of Parkinson's disease: a review of the evidence. Eur $\mathrm{J}$ Epidemiol. 2011;26:(Suppl 1):S1-58

47. Breckenridge CB, Berry C, Chang ET, Sielken RL Jr, Mandel JS. Association between Parkinson's disease and cigarette smoking, rural living, well-water consumption, farming and pesticide use: Systematic review and meta-analysis. PLoS ONE 11:e0151841. 